\title{
Clinicopathological Significance of Osteopontin in Cholangiocarcinoma Cases
}

\author{
Marut Laohaviroj ${ }^{1,2}$, Yaovalux Chamgramol ${ }^{1}$, Chawalit Pairojkul ${ }^{1}$, Jason \\ Mulvenna $^{3}$, Banchob Sripa ${ }^{1,2 *}$
}

\begin{abstract}
Cholangiocarcinoma (CCA) is generally a rare primary liver tumor of the bile duct with extremely poor clinical outcomes due to late diagnosis. Osteopontin (OPN) is the most abundant expressed gene in intrahepatic CCA and its involvement in tumor aggressiveness suggests it could be a useful prognostic biomarker. However, the prognostic significance of OPN expression in CCA is still controversial. We therefore immunohistochemically studied OPN expression in 354 resected CCAs and correlated the results with patient clinicopathological parameters. OPN expression was separately scored according to the percentage of cancer cells or degree of stromal tissue staining and classified as low (score 0-1) and high (score 2-3). OPN expression in CCA cells was found in 177 out of 354 patients $(56.5 \%)$, whereas stroma was positive in 185 out of 354 patients $(52.3 \%)$. Univariate analysis with several of the aforementioned parameters revealed that stromal but not cancer cell OPN expression was significantly associated with tumor size, tumor direct invasion into normal liver parenchyma, regional lymph node metastasis and higher staging. The combination of cancer cell and stromal OPN expression demonstrated a positive trend for linkage with lymph node metastasis. Multivariate analysis identified gender, the presence of lymphatic permeation and lymph node metastasis, but not OPN expression, as independent prognostic factors. This study confirms the presence of stromal OPN expression in tumor aggressiveness but not survival in CCA patients.
\end{abstract}

Keywords: Cholangiocarcinoma - osteopontin - biomarker - prognostic marker - immunohistochemistry

Asian Pac J Cancer Prev, 17 (1), 201-205

\section{Introduction}

Cholangiocarcinoma (CCA) is a fatal primary malignant tumor arising from the biliary tract epithelia either within the liver or extrahepatic. It is a major public health problem in northeastern Thailand and in Lao Peoples Democratic Republic (Lao PDR) where the carcinogenic liver fluke, Opisthorchis viverrini is endemic (Sripa et al., 2011). In the rest of the world, World Health Organization (WHO) have also reported a global increase in CCA related mortality in Asia, Europe, America and Australia (Khan et al., 2002). CCA grows slowly in the early stages but rapidly progresses and metastasizes aggressively in later stages. Patients usually present late clinically and the prognosis then is poor (Uttaravichien et al., 1999; Shaib and El-Serag, 2004). At present, there is no successful treatment and as patients typically present late, surgical resection is the only available treatment of choice (Uttaravichien et al., 1999). However, the mortality rate is still high with much less than 5 years survival in certain types of CCA (Khuntikeo et al., 2014). Therefore, biomarkers for earlier diagnosis and improved prognosis of this cancer are necessary (Ghouri et al., 2015).
Osteopontin (OPN) is a glyco-phosphoprotein found in the extracellular matrix of various tissues such as kidney, bone and teeth. OPN plays a role in a number of physiological and pathologic processes such as cell adhesion, migration, angiogenesis, apoptosis, inflammation and wound healing (Standal et al., 2004). Moreover, OPN is also involved in tumor development, progression, and metastasis and prognosis in several cancers (Subraman et al., 2015). OPN has been shown to be the most abundant expressed gene in intrahepatic CCA (Hass et al., 2008; Sulpice et al., 2013). Immunohistologically, OPN is found at the cellular apical surface, cytoplasm and also extracellular matrix of CCA tissues and a decreased expression in carcinoma cells (Terashi et al., 2004; Iguchi et al., 2009) or overexpression in stroma (Sulpice et al., 2013) was associated with tumor aggressiveness. However, the prognostic significance of OPN expression in CCA is still controversial.

Therefore, this study aimed to determine whether OPN expression both in carcinoma cells and stroma in resected specimens from CCA patients is associated with clinicopathological parameters and clinical outcomes in a large number of cases.

${ }^{1}$ Department of Pathology, ${ }^{2}$ WHO Collaborating Centre for Research and Control of Opisthorchiasis (Southeast Asian Liver Fluke Disease), Tropical Disease Research Laboratory, Faculty of Medicine, Khon Kaen University, Khon Kaen, Thailand, ${ }^{3}$ Infectious Disease and Cancer, QIMR Berghofer Medical Research Institute, Brisbane, QLD, Australia*For correspondence: banchob@ kku.ac.th 


\section{Materials and Methods}

\section{Tissue samples and ethics statement}

Tissue samples collected from 354 cholangiocarcinoma cases who underwent hepatectomy at Srinagarind Khon Kaen University Hospital filed at the Department of Pathology, Faculty of Medicine, Khon Kaen University, Khon Kaen, Thailand were used (Fedor and De Marzo, 2005). All tissue slides were histopathologically evaluated for histologic types, tumor direct invasion, and intrahepatic vascular, lymphatic and/or perineural invasion according to WHO classification (Nakanuma et al., 2010). Patient information regarding age, sex, gross types, size, regional lymph node metastasis, clinical staging, and survival was obtained from medical records of the Liver Fluke and Cholangiocarcinoma Research Center, Khon Kaen University. All human specimen collection and use in this study was approved by the Human Ethics Research Committee of Khon Kaen University (HE581073).

\section{Immunohistochemical staining}

Detection of OPN in CCA tissues was done by indirect immunohistochemistry (Yonglitthipagon et al., 2010). Briefly, 4- $\mu$ m-thick paraffin sections on silane-coated slides (Sigma Chemicals, USA) were dewaxed in a series of xylene and rehydrated in graded ethanol and distilled water, respectively. Antigen retrieval was done by high temperature technique using $10 \mathrm{mM}$ citrate buffer and a pressure cooker. Endogenous peroxidase activity was blocked in methanol with $3 \%$ hydrogen peroxide $\left(\mathrm{H}_{2} \mathrm{O}_{2}\right)$. Blocking for non-specific staining was performed by incubation with 5\% normal horse serum. OPN antibody (polyclonal, ab8448, Abcam, UK) at a dilution of 1:300 was applied onto the tissue slides overnight at $4^{\circ} \mathrm{C}$. The slides were then washed with PBS and incubated with secondary antibody (anti-rabbit IgG-HRP conjugated, ab97051, Abcam, USA) for $30 \mathrm{~min}$ at room temperature. After thorough washing, the slides were developed in DAB solution and counterstained with Mayer's hematoxylin, dehydrated, cleared and mounted. PBS was substituted for the primary antibody in the control sections. A positive stain was determined by dark brown color in the tissue sections.

\section{Scoring of OPN expression}

OPN expression in CCA tissues was semi-quantitatively scored according to the percentage of positive CCA cells in a tissue section as follow: a score of $0,1,2$ and 3 refer to positive CCA cells of $<10 \%, 11-33 \%, 34-66 \%$ and $67-100 \%$, respectively. For analysis, a score $0-1$ were defined as negative or low expression whereas a score of 2-3 were defined as positive or high expression. The stroma were semi-quantitatively scored according to the positive stromal staining as no, patchy, partial and diffuse staining (score 0, 1, 2 and 3, respectively). Similarly, the score 0 - 1 was defined as negative or low expression, and score 2 - 3 was defined as positive or high expression.

\section{Statistical analysis}

Data analysis was done using SPSS V.19.0 (IBM) statistical package and graphical images were generated by Prism 6.0 (GraphPad) software. For cross-sectional analyses, Chi-square and Chi-square for trend were used to analyze the relationship between a candidate protein expression and categorical clinicopathological parameters (e.g., age, gross type and tumor invasion). The KaplanMeier method was used for calculation of cumulative survival. Univariate analysis (log-rank test) was utilized for survival analysis between low expression and high expression groups. Multivariate analysis was done by Coxregression model. A P $<0.05$ was considered statistically significant.

\section{Results}

\section{OPN expression in CCA tissues}

OPN expression was observed in both CCA cells and stroma (Figure 1A-D). Normal bile duct epithelium and stromal tissue at the portal triad showed no, or slight, OPN expression (Figure 1A). For cancer cells, the expression was mainly localized in the cytoplasm or apical surface (Figure 1D). The stromal OPN expression ranged from negative (Figure 1B) to high expression (Figure 1C) in tumor tissue. Semi-quantitative grading of the OPN expression revealed positive cancer cells (grade 2-3) in 177 out of 354 cases $(56.5 \%)$. Stromal OPN expression (grade 2-3) was found in 185 out of 354 cases (52.26\%). No significant correlation between the expression of OPN

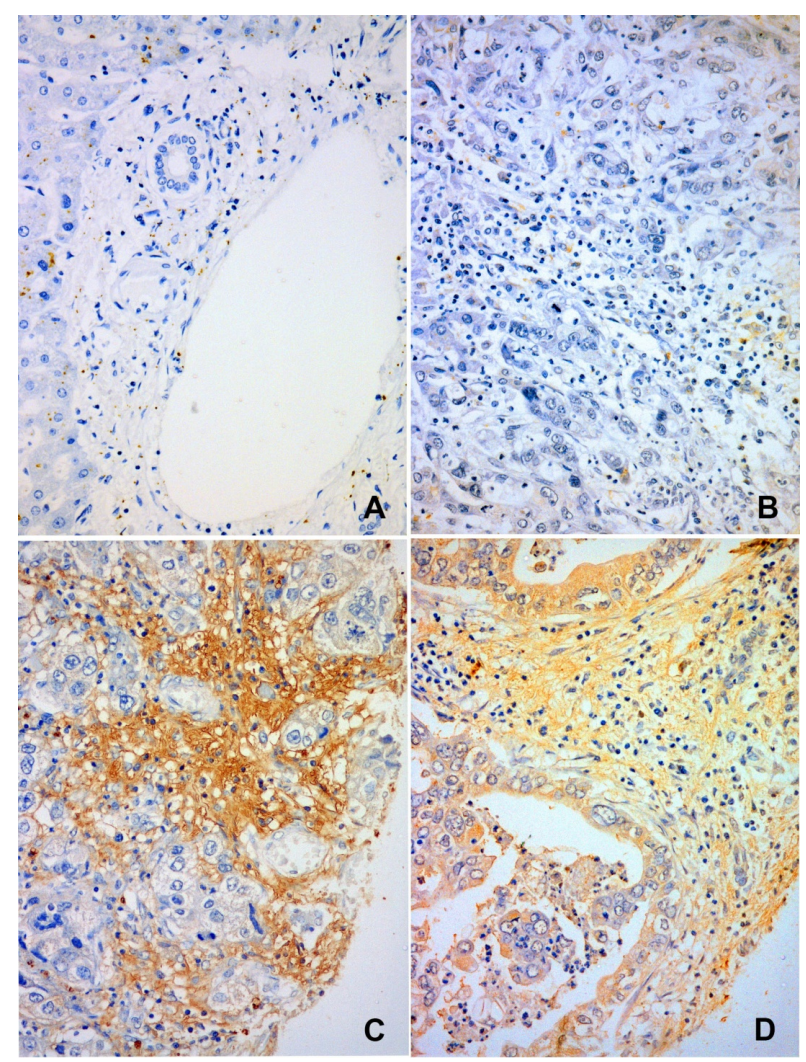

Figure 1. Immunohistochemical Staining of OPN in CCA Cells and Stromal Tissue. A) normal portal triads of the liver shows no OPN expression in bile duct epithelium and stroma, B) negative OPN in cancer cells and stroma, C) negative OPN in cancer cells but positive in stroma, and $D$ ) positive OPN expression in both cancer cells and stroma. Immunoperoxidase staining, original magnification $\times 100$ (A) and $\times 200$ (B, C and D) 

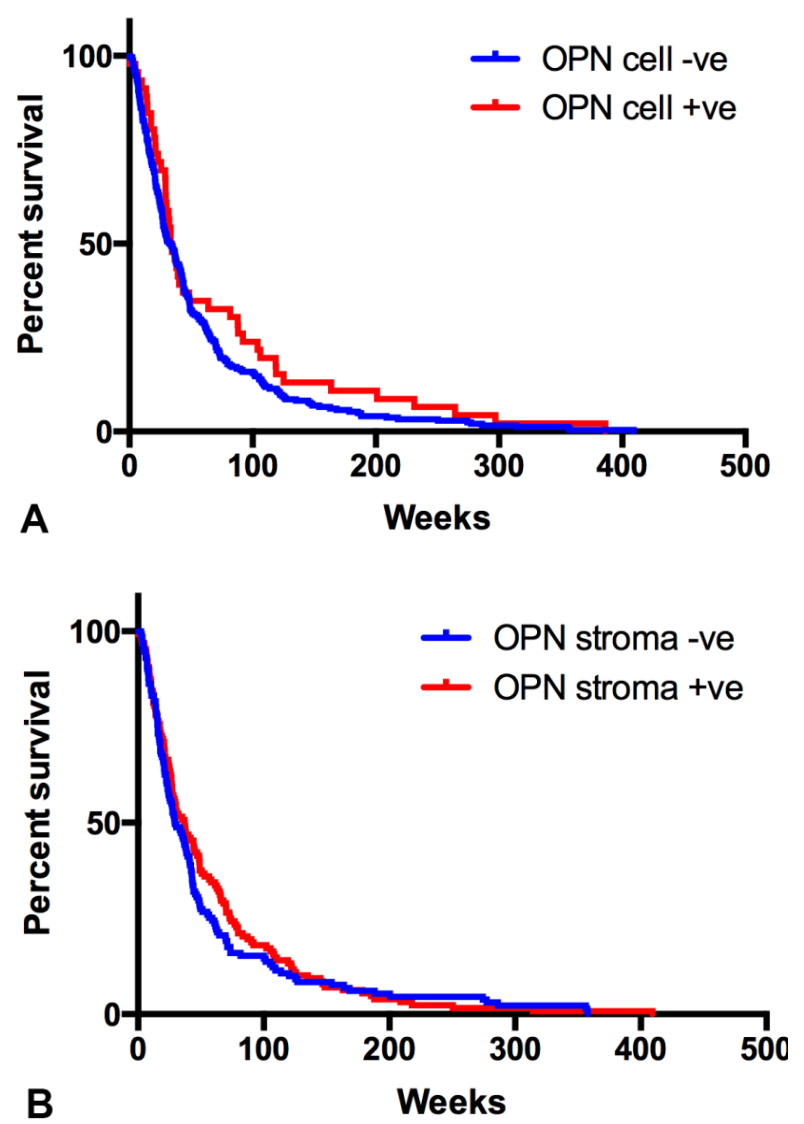

Figure 2. Kaplan-Meier Survival Analysis of OPN Expression in CCA Cells (A) and Stroma (B). OPN expression either in carcinoma cells or stroma shows no influence in patients' survival $(\mathrm{P}>0.05)$

in the cancer cells and stroma was found. OPN expression in cancer cells as stratified by low (grade 0-1) or high (grade 2-3) expression was not associated with any of the clinicopathological parameters studied (age, gender, histotype, gross type, tumor size, vascular invasion, lymphatic invasion, perineural invasion, tumor direct invasion, or, lymph node metastasis) as shown in Table 1. However, stromal expression of OPN was significantly associated with small tumor size $(\leq 5 \mathrm{~cm})$, tumor direct invasion into normal liver parenchyma, regional lymph node metastasis and higher staging (Table 1). Cases with positive OPN expression in either cancer cells or stromal or both showed a more potential to metastasize to regional lymph nodes $(\mathrm{P}<0.05)$. In addition, OPN expression in cancer cells and/or stroma has a positive trend to occur in higher staging (log-rank test for trend, $\mathrm{P}<0.05)$.

\section{OPN expression and overall survival}

Kaplan-Meier survival analysis revealed the median survival for patients with low OPN expression in cancer cells was not different from the higher ones $(33.57 \pm 3.54$ weeks vs $33.30 \pm 3.35$ weeks, respectively). Similarly, stromal OPN expression was not associated with overall survival (median survival $30.42 \pm 4.11$ weeks vs $34.57 \pm 3.87$ weeks for low and high expression, respectively) (Table 2 ). The survival curve of the patients created based on low and high OPN expression in CCA tissue is shown in Figure 2AB.

Twelve potential prognostic factors including age,
Table 1. Clinicopathological Variables and OPN Expression in Cancer Cells and Stroma in CCA Tissues

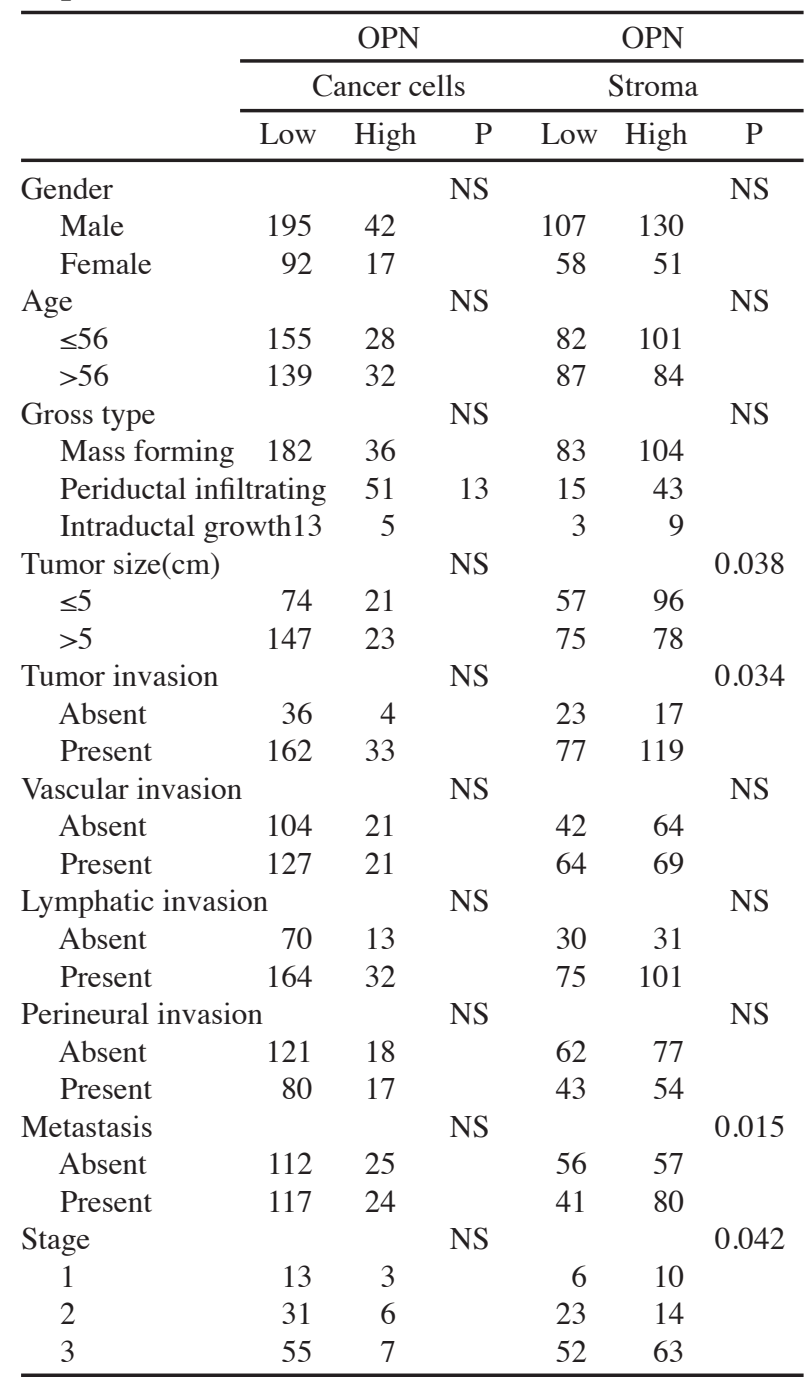

*NS $=$ No significant different. OPN grading $0-1=$ Low and 2-3 = High. The sum of subgroups does not match the totals due to unavailable or missing data

gender, histology group, gross type, tumor size, vascular invasion, lymphatic permeation, perineural invasion, tumor invasion, lymph node metastasis, and OPN expression were analyzed using univariate analysis (logrank test). It was found that gender, histology group, vascular invasion, lymphatic permeation, tumor direct invasion, lymph node metastasis but not OPN expression were significantly associated with patient survival (see Table 2). Multivariate analysis using Cox-regression model showed that gender and the presence of lymphatic permeation and lymph node metastasis were identified as independent prognostic factors (Table 2).

\section{Discussion}

Cholangiocarcinoma is a rare cancer worldwide but it is a prevalent cancer in liver fluke endemic areas in Southeast Asia (Shin et al., 2010; Sripa et al., 2010). CCA is one of the most fatal cancers with poor prognosis due to typically late diagnosis (Khuntikeo et al., 2014). Numerous conventional prognostic markers, such as gross types, histologic types, tumor staging, lymph node 


\begin{tabular}{|c|c|c|c|c|c|}
\hline & \multicolumn{5}{|c|}{ Overall Survival } \\
\hline & \multicolumn{2}{|c|}{$\begin{array}{l}\text { Univariate analysis } \\
\text { (Log-rank) }\end{array}$} & \multicolumn{3}{|c|}{$\begin{array}{l}\text { Multivariate analysis } \\
\text { (Cox regression) }\end{array}$} \\
\hline & Median time, $w k \pm S E$ & $\mathrm{p}$ & Hazard ratio & $95 \% \mathrm{CI}$ & $\mathrm{p}$ \\
\hline Gender & & 0.04 & & & 0.011 \\
\hline Male & $29.00 \pm 1.99$ & & 1 & & \\
\hline Female & $40.14 \pm 4.01$ & & 0.66 & $0.48-0.91$ & \\
\hline Age & & NS & & & NS \\
\hline$\leq 56$ & $35.00 \pm 4.12$ & & 1.10 & $0.82-1.48$ & \\
\hline$>56$ & $30.00 \pm 3.70$ & & 1 & & \\
\hline Gross type & & NS & & N/A & \\
\hline Mass forming & $32.00 \pm 3.29$ & & & & \\
\hline Periductal infiltrating & $32.29 \pm 8.08$ & & & & \\
\hline Intraductal growth & $87.90 \pm 14.61$ & & & & \\
\hline Tumor size & & NS & & NS & \\
\hline$\leq 5 \mathrm{~cm}$ & $36.70 \pm 2.96$ & & 0.97 & $0.72-1.31$ & \\
\hline$>5 \mathrm{~cm}$ & $29.57 \pm 2.95$ & & 1 & & \\
\hline Tumor direct invasion & & 0.02 & & & \\
\hline Absent & $37.14 \pm 4.90$ & & 1 & & \\
\hline Present & $27.42 \pm 1.66$ & & 1.40 & $0.94-2.08$ & \\
\hline Vascular invasion & & 0.04 & & & NS \\
\hline Absent & $37.14 \pm 3.81$ & & 1 & & \\
\hline Present & $25.42 \pm 1.99$ & & 1.11 & $0.82-1.52$ & \\
\hline Histology group & & 0.03 & & & NS \\
\hline Less diff. & $29.00 \pm 2.63$ & & 1 & & \\
\hline Well diff. & $41.14 \pm 6.47$ & & 0.80 & $0.59-1.08$ & \\
\hline Lymphatic permeation & & 0.001 & & & 0.004 \\
\hline Absent & $38.14 \pm 3.06$ & & 1 & & \\
\hline Present & $25.42 \pm 1.95$ & & 1.67 & $1.17-2.39$ & \\
\hline Perineural invasion & & NS & & & NS \\
\hline Absent & $29.57 \pm 4.40$ & & 1 & & \\
\hline Present & $29.28 \pm 2.34$ & & 1.06 & $0.77-1.45$ & \\
\hline Lymph node metastasis & & 0.033 & 0.037 & & \\
\hline Absent & $32.14 \pm 6.31$ & & 1 & & \\
\hline Present & $26.57 \pm 4.09$ & & 1.37 & $1.01-1.85$ & \\
\hline OPN expression in cancer cells & & NS & & & NS \\
\hline Absent & $33.30 \pm 3.35$ & & 1 & & \\
\hline Present & $33.57 \pm 3.54$ & & 0.79 & $0.52-1.19$ & \\
\hline OPN expression in stroma & & NS & & & NS \\
\hline Absent & $30.42 \pm 4.11$ & & 1 & & \\
\hline Present & $34.57 \pm 3.87$ & & 0.87 & $0.64-1.17$ & \\
\hline
\end{tabular}

$* \mathrm{CI}=$ Confident interval, NS $=$ No significant different, N/A = Not applicable, diff $=$ differentiate

metastasis (Sriputtha et al., 2013), serum biomarkers (Silsirivanit et al., 2011; Grunnet and Mau-Sorensen, 2014) and specific proteins/molecular markers (Juntavee et al., 2005; Sirica et al., 2009; Huang et al., 2012) have been described. For OPN, its prognostic significance is still controversial (Terashi et al., 2004; Iguchi et al., 2009; Sulpice et al., 2013). Here we reported in a large number of CCA cases that expression of OPN in tumorous stroma and/or cancerous cells was associated with direct tumor invasion, lymphatic permeation and lymph node metastasis but was not associated with prognosis in CCA patients.

OPN is a secreted glycoprotein found in the extracellular matrix both in normal and cancerous tissues (Subraman et al., 2015). OPN plays roles alongside its receptor, CD44 and is involved in tumor invasion and metastasis in several cancer types including CCA (Bourguignon et al., 1999; Irby et al., 2004; Macri et al., 2009; Zhao et al., 2011; Sulpice et al., 2013; Qiu et al., 2014). Our study clearly showed that stromal OPN expression was associated with tumor size, direct tumor invasion, lymph node metastasis and higher staging similar to a previous report (Sulpice et al., 2013). In addition, we found new information about OPN over-expression in CCA cells and that stromal expression was associated with lymph node metastasis and high staging $(\mathrm{P}<0.05)$. This finding contradicts the reports of Terachi et al. (2004) and Iguchi et al. (2009) who described a decreased expression of OPN in carcinoma cells was linked tumor invasion and poor prognosis. However, their reports did not mention stromal OPN expression. Our findings from a larger number of cases complement and begin to fill in the gaps from these three earlier publications.

Survival analysis using both univariate and multivariate analysis, did not find a significant association between cancerous or stromal OPN expression and survival time of the CCA patients even though our stromal or cancerous OPN expression was associated with some tumor aggressive characteristics. Other factors may play more important roles in patient survival. In our study, lymphatic 
permeation and regional lymph node metastasis were independent prognostic markers identified by multivariate analysis. Similarly, even decreased OPN expression in CCA cells was significantly associated with poor survival by univariate analysis but failed to sustain this significance in multivariate model (Iguchi et al., 2009), unlike Sulpice et al. (2013) who demonstrated that stromal OPN expression was an independent prognostic marker.

In conclusion, our study has provides more information on OPN expression both in cancer cells and stroma of CCA tissue. The expression of OPN in CCA tissue again was found to have a positive association with tumor aggressiveness as described in several earlier studies. However, in a number of CCA cases studied, we did not find any association between OPN expression and patient survival.

\section{Acknowledgements}

This work was partially supported by the Thailand Research Fund (TRF) (RTA 5680006), the National Cancer Institute (R01CA155297) and National Institute of Allergy and Infectious Diseases (NIAID) (P50AI098639), NIH. Marut Laohaviroj is a Royal Golden Jubilee PhD Scholar (PHD/0301/2552) under the supervision of Professor Banchob Sripa. BS is a TRF Senior Research Scholar. The content is solely the responsibility of the authors and does not necessarily represent the official views of the NCI, NIAID or the NIH or the funders. Associate Professor John F. Smith is gratefully acknowledged for his kind assistance in English editing.

\section{References}

Bourguignon LY, Zhu H, Shao L, et al (1999). Rho-kinase (ROK) promotes $\mathrm{CD} 44 \mathrm{v}(3,8-10)$-ankyrin interaction and tumor cell migration in metastatic breast cancer cells. Cell Motil Cytoskeleton, 43, 269-87.

Fedor HL, De Marzo AM (2005). Practical methods for tissue microarray construction. Methods Mol Med, 103, 89-101.

Ghouri YA, Mian I, Blechacz B (2015). Cancer review: Cholangiocarcinoma. J Carcinog, 14, 1.

Grunnet M, Mau-Sorensen M (2014). Serum tumor markers in bile duct cancer--a review. Biomarkers, 19, 437-43.

Hass HG, Nehls O, Jobst J, et al (2008). Identification of osteopontin as the most consistently over-expressed gene in intrahepatic cholangiocarcinoma: detection by oligonucleotide microarray and real-time PCR analysis. World J Gastroenterol, 14, 2501-10.

Huang Q, Liu L, Liu CH, et al (2012). Expression of Smad7 in cholangiocarcinoma: prognostic significance and implications for tumor metastasis. Asian Pac J Cancer Prev, 13, 5161-5.

Iguchi T, Yamashita N, Aishima S, et al (2009). A comprehensive analysis of immunohistochemical studies in intrahepatic cholangiocarcinoma using the survival tree model. Oncol, 76, 293-300.

Irby RB, McCarthy SM, Yeatman TJ (2004). Osteopontin regulates multiple functions contributing to human colon cancer development and progression. Clin Exp Metastasis, 21, 515-23.

Juntavee A, Sripa B, Pugkhem A, et al (2005). Expression of sialyl Lewis(a) relates to poor prognosis in cholangiocarcinoma.
World J Gastroenterol, 11, 249-54.

Khan SA, Taylor-Robinson SD, Toledano MB, et al (2002). Changing international trends in mortality rates for liver, biliary and pancreatic tumours. J Hepatol, 37, 806-13.

Khuntikeo N, Pugkhem A, Titapun A, et al (2014). Surgical management of perihilar cholangiocarcinoma: a Khon Kaen experience. J Hepatobiliary Pancreat Sci, 21, 521-4.

Macri A, Versaci A, Lupo G, et al (2009). Role of osteopontin in breast cancer patients. Tumori, 95, 48-52.

Nakanuma Y, Sato Y, Harada K, et al (2010). Pathological classification of intrahepatic cholangiocarcinoma based on a new concept. World J Hepatol, 2, 419-27.

Qiu Y, Hu Y, Zhang ZY, et al (2014). Genetic association of osteopontin (OPN) and its receptor CD44 genes with susceptibility to Chinese gastric cancer patients. J Cancer Res Clin Oncol, 140, 2143-56.

Shaib Y, El-Serag HB (2004). The epidemiology of cholangiocarcinoma. Semin Liver Dis, 24, 115-25.

Shin HR, Oh JK, Masuyer E, et al (2010). Comparison of incidence of intrahepatic and extrahepatic cholangiocarcinoma--focus on East and South-Eastern Asia. Asian Pac J Cancer Prev, 11, 1159-66.

Silsirivanit A, Araki N, Wongkham C, et al (2011). A novel serum carbohydrate marker on mucin 5AC: values for diagnostic and prognostic indicators for cholangiocarcinoma. Cancer, 117, 3393-403.

Sirica AE, Dumur CI, Campbell DJ, et al (2009). Intrahepatic cholangiocarcinoma progression: prognostic factors and basic mechanisms. Clin Gastroenterol Hepatol, 7, 68-78.

Sripa B, Bethony JM, Sithithaworn P, et al (2011). Opisthorchiasis and Opisthorchis-associated cholangiocarcinoma in Thailand and Laos. Acta Trop, 120, 158-68.

Sripa B, Kaewkes S, Intapan PM, et al (2010). Food-borne trematodiases in Southeast Asia epidemiology, pathology, clinical manifestation and control. Adv Parasitol, 72, 305-50.

Sriputtha S, Khuntikeo N, Promthet S, et al (2013). Survival rate of intrahepatic cholangiocarcinoma patients after surgical treatment in Thailand. Asian Pac J Cancer Prev, 14, 1107-10.

Standal T, Borset M, Sundan A (2004). Role of osteopontin in adhesion, migration, cell survival and bone remodeling. Exp Oncol, 26, 179-84.

Subraman V, Thiyagarajan M, Malathi N, et al (2015). OPN -Revisited. J Clin Diagn Res, 9, 10-3.

Sulpice L, Rayar M, Desille M, et al (2013). Molecular profiling of stroma identifies osteopontin as an independent predictor of poor prognosis in intrahepatic cholangiocarcinoma. Hepatol, 58, 1992-2000.

Terashi T, Aishima S, Taguchi K, et al (2004). Decreased expression of osteopontin is related to tumor aggressiveness and clinical outcome of intrahepatic cholangiocarcinoma. Liver Int, 24, 38-45.

Uttaravichien T, Bhudhisawasdi V, Pairojkul C, et al (1999). Intrahepatic cholangiocarcinoma in Thailand.J Hepatobiliary Pancreat Surg, 6, 128-35.

Yonglitthipagon P, Pairojkul C, Chamgramol Y, et al (2010). Up-regulation of annexin A2 in cholangiocarcinoma caused by Opisthorchis viverrini and its implication as a prognostic marker. Int J Parasitol, 40, 1203-12.

Zhao XQ, Dong JH, Zhang WZ, et al (2011). Prognosis of ampullary cancer based on immunohistochemical type and expression of osteopontin. Diagn Pathol, 6, 98. 